\title{
Case Report: Repetitive Arm Movements During Sleep: A Polysomnographic Assessment
}

\author{
Mohammad Torabi-Nami ${ }^{1,2,3^{*}}$, Samrad Mehrabi ${ }^{2,4}$, Sabri Derman $^{5}$
}

1. Department of Neuroscience, School of Advanced Medical Sciences and Technologies, Shiraz University of Medical Sciences, Shiraz, Iran

2. Sleep Disorders Laboratory, Namazi Hospital, Shiraz University of Medical Sciences, Shiraz, Iran.

3. Shiraz Neuroscience Research Center, Shiraz University of Medical Sciences, Shiraz, Iran.

4. Division of Pulmonology, Department of Internal Medicine, School of Medicine, Shiraz University of Medical Sciences, Shiraz, Iran

5. Sleep Disorders Unit, American Hospital, Koç Foundation, Istanbul, Turkey.

Citation: Torabi-Nami, M., Mehrabi, S., \& Derman, S. (2016). Repetitive arm movements during sleep: A polysomnographic assessment. Basic and Clinical Neuroscience, 7(3), 269-275. http://dx.doi.org/10.15412/J.BCN.03070312

: http://dx.doi.org/10.15412/J.BCN.03070312

Article info:

Received: 24 July 2015

First Revision: 18 August 2015

Accepted: 21 December 2015

Key Words:

Polysomnography, Periodic arm movements during sleep, Sleep disorders

\begin{abstract}
A B S T R A C T
Sleep-related movement disorders should be differentiated from parasomnias, sleep-associated behavioral disorders, and epilepsy. Polysomnography (PSG) is the gold standard in evaluating such disorders. Periodic leg movement disorder during sleep (PLMS), hypnic jerks, bruxism, rhythmic movement disorder, restless legs syndrome, and nocturnal leg cramps have broadly been discussed in the literature. However, periodic arm movement disorder in sleep (PAMS) is a less-appreciated entity perhaps because arm surface electromyography is not an integral part of the standard polysomnography. Results from our PSG study in a case suspected for PAMS prompted us to herewith discuss this problem.
\end{abstract}

\section{Introduction}

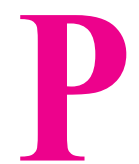

eriodic leg movements during sleep (PLMS) and restless-leg syndrome (RLS) are among the well-recognized clinical phenomena mainly by clinicians involved in the practice of sleep medicine (Karatas, 2007; Portaluppi, Cortelli, Buonaura, Smolensky, \& Fabbian 2009; Trotti et al., 2009; Valko, Siccoli, \& Bassetti, 2009; Torabi-Nami, 2012). The term periodic arm movements in sleep (PAMS) is however less appreciated partly because surface arm electromyography (EMG) is not routinely obtained in polysomnographic assessment (Yokota, Shiojiri, \& Hirashima, 1995). Having encountered a case suspected of PAMS, we recorded arm
EMG as a part of his full polysomnographic assessment. The present case discussion tries to highlight the clinical significance of PAMS as a subtype of sleep-related movement disorders.

\section{Case Presentation}

\subsection{Presenting Illness}

N.K. was a 58-year-old right-handed male complaining of difficulty in sleeping, heavy snoring, witnessed apneas, frequent nocturnal awakenings with daytime sleepiness, and fatigue since a year prior to presentation. He had tolerated sleeping complaints until 3 months ago while started to feel progressively unable to sleep supine

* Corresponding Author:

Mohammad Torabi-Nami, MD, PhD

Address: Department of Neuroscience, School of Advanced Medical Sciences and Technologies, Shiraz University of Medical Sciences, Shiraz, Iran.

Tel: +98 (71) 32317523

Fax: +98 (71) 32318042

E-mail: torabinami@sums.ac.ir 
due to electric-like throbbing sensations in his chest and upper limb. His wife reported the patient's vigorous arm and leg movements during sleep making it hard for her sharing bed with him. His usual bedtime was 10 PM; falling asleep quickly, getting out of bed at $5 \mathrm{AM}$. While he felt temporarily refreshed in the morning, he demanded much coffee to retain his daytime energy. However, despite drinking 8-10 cups of coffee a day, his Epworth Sleepiness Scale score was 12.

The patient reported neither the symptoms of RLS, complex behaviors such as speaking, yelling, rocking nor other sleep-related rhythmic movements, such as hypnagogic hallucinations, sleep paralysis, and cataplexy. He also complained of a 6-month history of neck and right shoulder pain with spasms radiating to his right arm and elbow with mild stiffness in the morning. He had been retired from a job at a car factory which involved repetitive arm movements in his duties.

\subsection{Clinical history and assessments}

Medical history was positive for hypertension but otherwise insignificant. He was non-smoker and denied any substance abuse. On examination, vital signs were within normal range and body mass index (BMI) was $25.7 \mathrm{~kg} /$ $\mathrm{m}^{2}$. Oronasal examination revealed nasal congestion and class 3 Mallampati score. Paravertebral muscle spasm was not present and examination revealed normal range of motion in all joints without any erythema or swelling. Neurological exam was unremarkable except for sensory deficit to pin-prick and light touch in the forearm, thumb, and index finger on the right side. His deep-tendon reflexes were depressed in his right arm.
His current medications included diclofenac sodium SR $100 \mathrm{mg}$ qhs, depakine $500 \mathrm{mg}$ bid, sertraline $50 \mathrm{mg}$ qd, clonazepam $0.5 \mathrm{mg}$ qhs, tamsulosin $0.4 \mathrm{mg}$ qd, amlodipine qd, and losartan $25 \mathrm{mg}$ qd.

EMG-NCV revealed no remarks while MRI showed $\mathrm{C} 3$ to $\mathrm{C} 7$ nerve roots impingement and canal stenosis. Brain MRI was normal. The basic metabolic panel, B12 and thyroid tests were normal. His ferritin level was 48 . He had almost no restful sleep over the last 3 months prior to present assessment.

To further investigate the patient's sleep complaints, he was referred to our hospital-based sleep unit for fullnight polysomnographic study.

\subsection{Polygraphic study}

Having an informed written-consent signed by the patient (enabling diagnostic, therapeutic, and research use of the test data), all-night polysomnographic sleep study was performed at our sleep disorders laboratory, between 10:40 PM and 6:40 AM the next morning. The SOMNOscreen system and the DOMINO analysis software were used for the recording and analysis of the sleep data. A technician was present during the entire recording. The following data were documented to a special sleep recording computer and scored according to the latest American Academy of Sleep Medicine manual for sleep scoring and analysis: EEG (F3-A2, F4-A1, C3-A2, C4-A1, O1-A2 and O1-A2), EOG (right), EOG (left), EMG (submental), EMG (right forearm on extensor carpi radialis and left leg on anterior tibialis), breathing effort (chest and abdomen), air intake (mouth/nose air flow), snoring sounds, oxygen saturation, plethysmo-

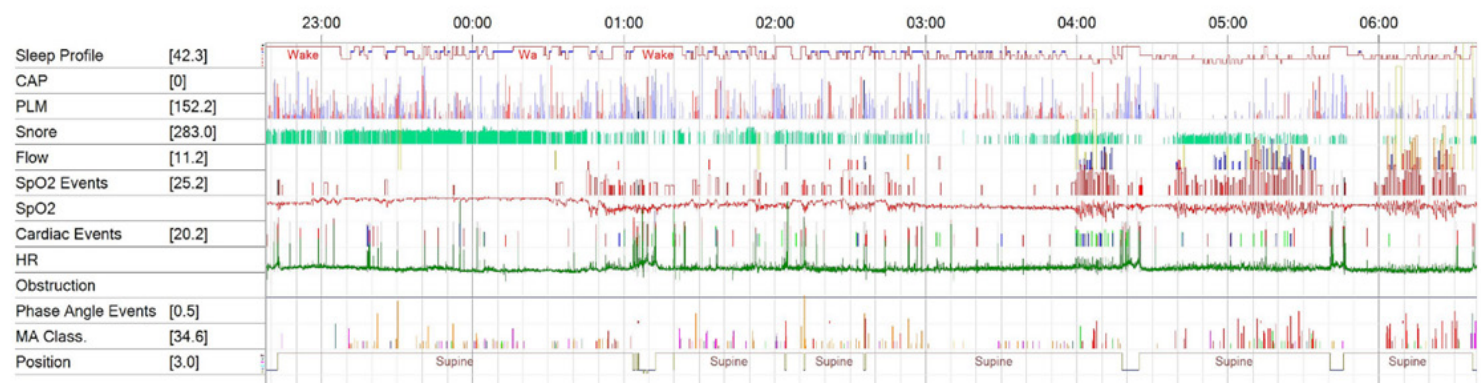

NEUR:SCIENCE

Figure 1. Sleep profile and summary of events in polysomnographic assessment of the patient. As demonstrated, except for airflow limitations and $\mathrm{O}_{2}$ desaturation, much of the events including periodic limb movements were predominately evident in the first half of the recording. The study revealed rapid stage transition and unfavorable sleep cycle integrity as well as repeated awakenings after sleep onset. CAP: cyclic alternating patterns, PLM: periodic limb movements, HR: heart rate, MA: microarousal. 


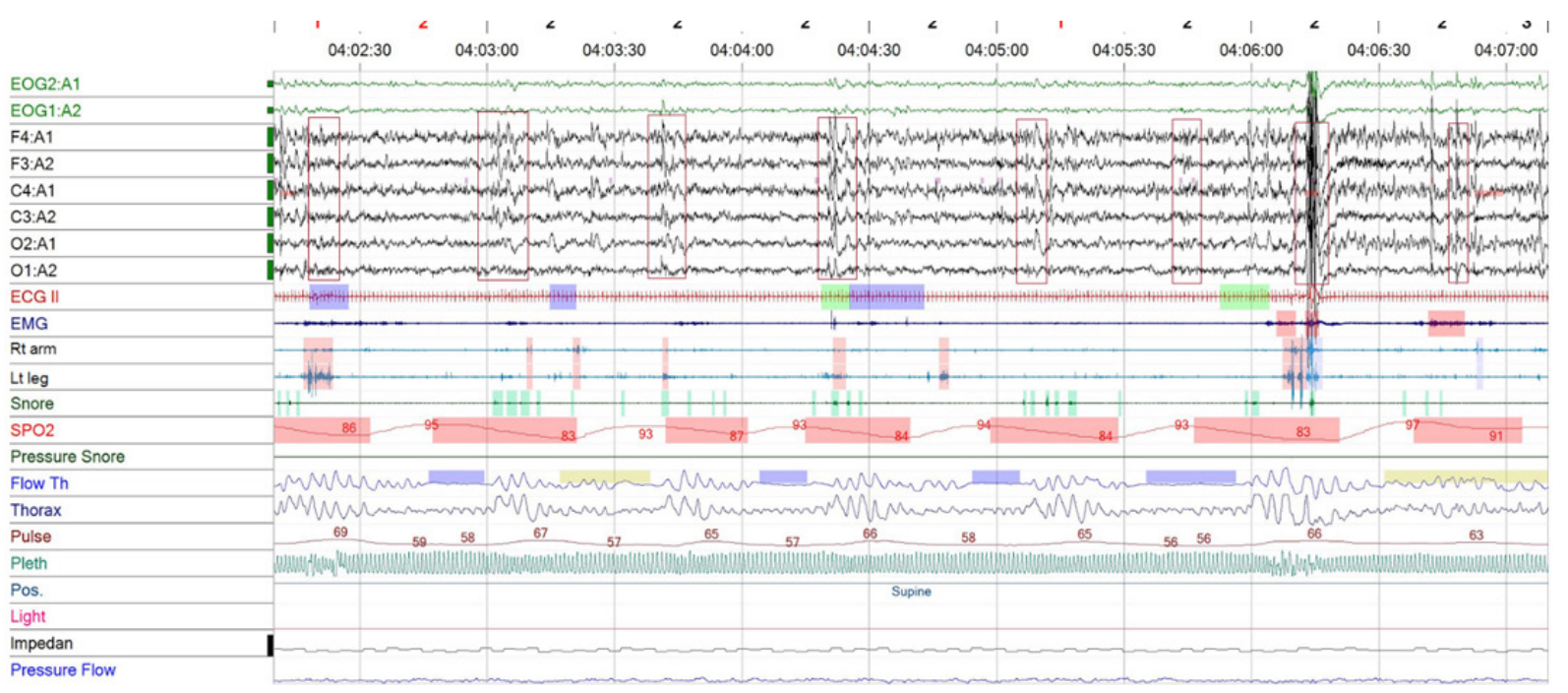

NEUR:SCIENCE

Figure 2. Sleep-related respiratory events: A 300-second epoch from the patient's PSG study representing samples of obstructive sleep apneas and airflow limitations during N2 sleep resulting in significant desaturation. Red boxes in EEG indicate $\mathrm{O}_{2}$ desaturation-related and apnea-induced microarousals. These sleep-related microarousals were however not abundant in this case. In the event of moderate to severe obstructive sleep apnea/hypopnea syndrome, the sleep disordered breathing may significantly impair the quality and integrity of sleep (Torabi-Nami et al., 2015). EOG: electrooculography, F: Frontal, C: central, O: occipital, ECG: electrocardiography, EMG: electromyography, Th: thermistor, pleth: plethysmography, pos: position.

graph, ECG, heart rate, and sleeping position (Berry et al., 2012; Grigg-Damberger, 2012).

\section{Results}

His polysomnographic study revealed periodic limb movements during sleep as well as apnea-hypopnea events resulting in desaturation microarousals. The patient had 35 obstructive, 18 central and 16 mixed type apneas, lasting 21 seconds on average during the sleep period. Apnea/hypopnea index (AHI) was 11.7 (number of apnea/hypopnea events per hour of sleep; Normal $\leq 5$ ). There were 13 flow limitations in non-rapid eye movement (NREM) sleep associated with loud snores and gasping sounds. His baseline oxygen saturation was $94 \%$. SpO2 dropped 158 times to more than 4\%. There were 130 desaturation below $90 \%$ and only one below $80 \%$. The lowest $\mathrm{O}_{2}$ saturation recorded value was $79 \%$. The patient's average heart rate in sleep was $57 \pm 8 \mathrm{bpm}$. In the absence of significant tachycardia or bradycardia, the patient had 43 premature ventricular contractions during the recording.

The attending technician observed mild to moderate degree of episodic snoring (PSG revealed $\mathrm{N}=1554$, over $22.6 \%$ of the sleeping time). The patient's sleep quality was below average. During 481 minutes of recording time, the patient slept for 352 minutes after 4 minutes of initial sleep latency (REM intrusion). Sleep efficacy was $73.3 \%$ (normal $\geq 90 \%$ ).
The patient had a moderate increase in the amount of light sleep (stage $\mathrm{N} 1=22.8 \%$ ), mildly increased stage 2 sleep (N2=49.4\%), normal percentage of rapid eye movement (REM) sleep ( $\mathrm{R}=24.5 \%)$ and markedly decreased deep sleep $(\mathrm{N} 3=3.3 \%)$. There were abundant spindles and cyclic alternating patterns (suggesting arousal instability) in $\mathrm{N}_{2}$. Very short REM latency and reversed distribution of REM periods (REM rebound) are mostly attributed to chronic sleep deprivation. The patient had frequent WASO (wakes after sleep onset) lasting for more than 60 seconds $(\mathrm{N}=41)$ and 197 microarousals $(\mathrm{N}=0$ on EEG lasting for less than 30 seconds) mostly during NREM. About $29.9 \%$ of the microarousals were related to periodic limb movement disorder during sleep and 30\% with desaturation (Figures 1 and 2).

EMG recording was significant for periodic leg movement disorder in sleep (PLMS). The diagnostic PSG showed 505 isolated periodic limb movements of sleep (PLMS) with a periodic limb movement (PLM) index of 66 per hour; 392 were associated with arousals with a resulting PLM arousal index of 52 per hour. Periodic arm movements dominated the first half of the night, whereas the second half revealed a mixed picture of arm and leg movements (Table 1 and Figure 3).

\section{Diagnosis, Treatment, and Follow up}

All-night polysomnographic results indicated inefficient sleep with repeated awakenings after sleep onset 
Table 1. PLMS, including periodic leg and arm movements during sleep. The PAMS index of 43.1 indicated the number of right arm movements per hour during sleep. As outlined in the table, respiratory- and body position-related limb movements were quite infrequent as compared to isolated events.

\begin{tabular}{|c|c|c|c|c|c|}
\hline \multicolumn{7}{|c|}{ Periodic Limb Movements during Sleep } \\
\hline & Sleep & REM & Non-REM & Wake & Total \\
\hline Isolated limb movements (Index) & $505(66)$ & $135(93.6)$ & $370(71.1)$ & $369(172.3)$ & $874(102.2)$ \\
\hline Periodic arm movements (Index) & $253(43.1)$ & $68(47.2)$ & $185(41.7)$ & $132(61.6)$ & $385(48.0)$ \\
\hline Resp-limb movement (Index) & $2(0.3)$ & - & $2(0.5)$ & - & $2(0.2)$ \\
\hline Body position-limb movements (Index) & $1(0.2)$ & $1(0.7)$ & - & $13(6.1)$ & $14(1.7)$ \\
\hline PLMS with microarousal (Index) & $392(52)$ & $121(18.7)$ & $271(33.5)$ & - & $392(52)$ \\
\hline
\end{tabular}

NEUR SCIENCE

and significant loss of deep sleep. Patient's sleep insufficiency is mostly related to periodic arm movements during sleep (PAMS)/PLMS and to lesser extent, respiratory issues causing significant desaturation-arousals during sleep. Frequent microarousals were reflected in decreased pulse transit time (PTT). A color-coded brain map using quantitative EEG has reflected microarousals concurrently occurred with an isolated right arm movement (Figure 4).

Accordingly, the following diagnoses were made: 1) Periodic arm movements of sleep (PAMS); 2) Periodic leg movements of sleep (PLMS); 3) Upper airway resistance syndrome; 4) Neck pain; and 5) Caffeine abuse.

To treat the mentioned problems, the following treatments and recommendations were prescribed: 1) The isolated microarousals and decreased PTT were possibly related to mechanical pain (cervical nerve roots impingement). Therefore, pain management under the care of physical medicine and rehabilitation expert is suggested. 2) To control PAMS as the main cause of impaired sleep in this patient, Pramipexole $0.18 \mathrm{mg}$ po qd (7 PM) for 1 week then $0.35 \mathrm{mg}$ po qd (7 PM) for 7 weeks was suggested. 3) Pregabalin $200 \mathrm{mg}$ po qhs (10 PM). 4) Clonazepam dose increased to $1 \mathrm{mg}$ qhs for 3 weeks and sleep physician visit for possible taper-off was suggested. 5) Tight sleep hygiene measures (reducing amount of evening meals with about 3-4 hours between meals and sleeping time as well as avoiding the use of caffeine at night) were suggested. The patient was informed of caffeine's negative impact on sleep quality and advised to reduce its consumption as much as possible. 6) Nocturnal $\mathrm{O}_{2}$ supplementation through nasal prongs at $3 \mathrm{~L} / \mathrm{m}$ for 4 weeks, and follow up visit thereafter was recommended.

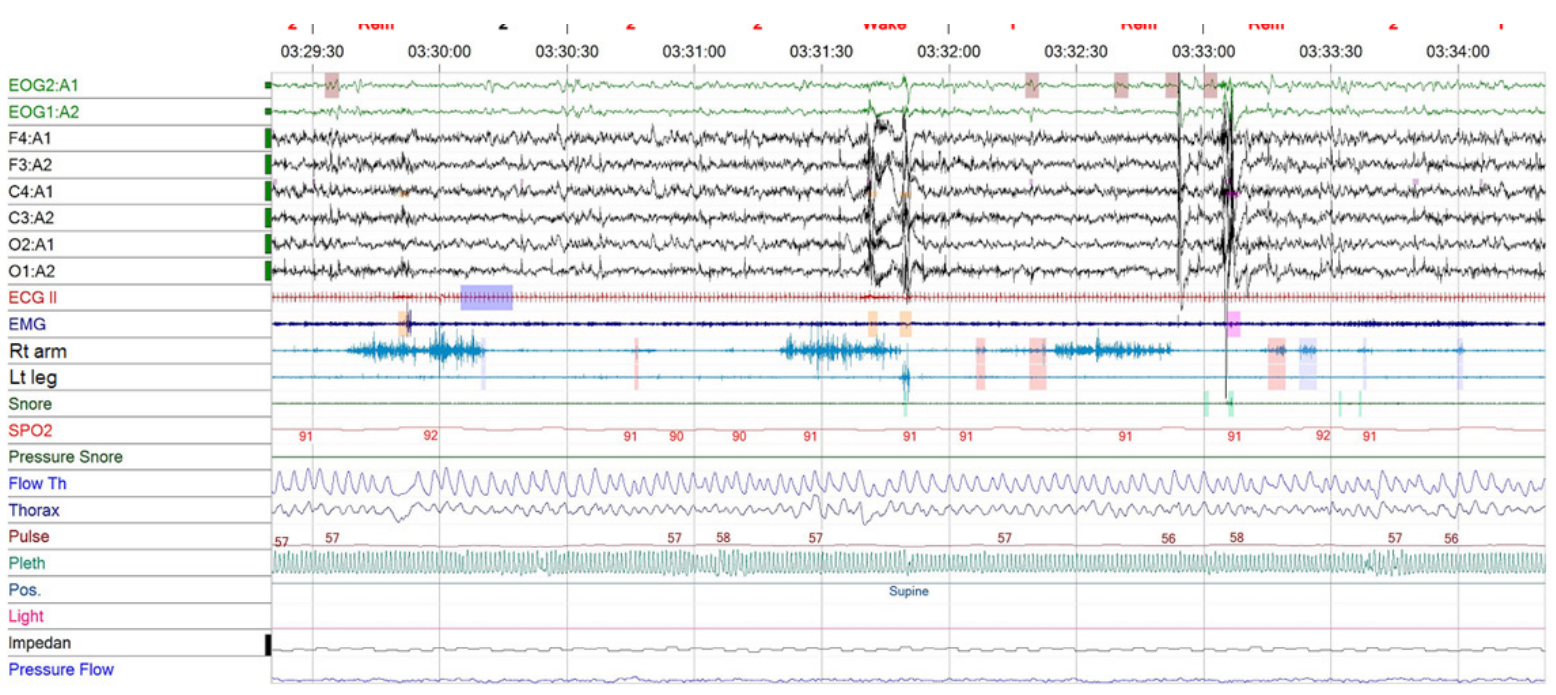

NEUR:SCIENCE

Figure 3. A 300-second epoch of the patient's PSG study showing right arm periodic movements in N2 and REM sleep resulting in awakening and microarousal, respectively. Arm movements are isolated from respiratory events, flow limitation or $\mathrm{O}_{2}$ desaturation. EOG: electrooculography, F: Frontal, C: central, O: occipital, ECG: electrocardiography, EMG: electromyography, Th: thermistor, Pleth: plethysmography, Pos: position. 


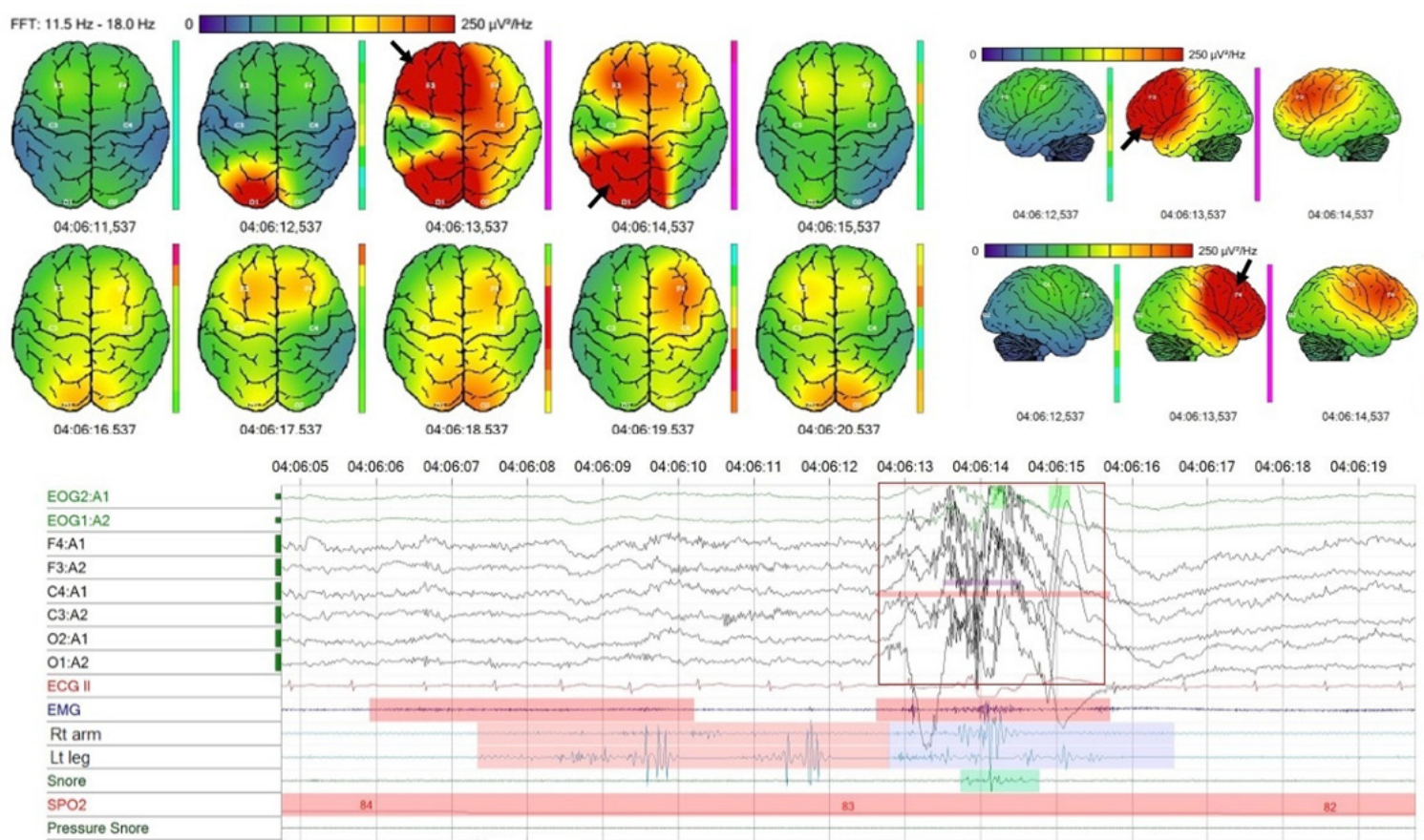

NEUR:SCIENCE

Figure 4. The color-coded quantitative EEG brain map using fast Fourier transform (FFT) method (Farabi et al., 2014) to isolate beta-frequency intrusions during N2 sleep. Hot colors represent fast frequencies intruded into NREM sleep resulting in a microarousal (4:06:13 and 4:06:14) concurrently occurred with an isolated right arm movement (blue marking). In N2 sleep, the EEG is generally dominated by alpha and theta activities, often interpreted as light sleep by the neural network. This plot demonstrates a typical PAMS-related microarousal with frontal (F3) and occipital (O1) beta-wave intrusions shown in red (arrows). Treating PAMS and preventing desaturation-related microarousals are expected to decrease such microarousals episodes.

The follow-up evaluation in sleep clinic for 4 weeks after medical therapy suggested a notable subjective improvement in sleep quality and significantly reduced abnormal movement reported by the patient's spouse as well.

\section{Discussion}

This particular case demonstrates that PAMS is a relatively uncommon phenomenon encountered during sleep recording (Yokota, Shiojiri, \& Hirashima, 1995). Sleep specialists are more familiar with PLMS than PAMS. This might be firstly due to lack of arm EMG recording, and secondly arm movements tend to be not as prominent as those of legs and their amplitude is not as robust (Janssen, Harlaar, \& de Groot, 2015). As such, knowledge about PAMS has remained scant. This specific presentation prompted us to tap into PAMS as a subtype of sleep-related movement disorders.

PAMS has been reported in a handful number of reports (Yokota, Shiojiri, \& Hirashima, 1995; Zucconi et al., 2006; Walters et al., 2007; Hoque \& Chesson, 2010; Malangre, Leinen, \& Blischke, 2014). Given the unclear underlying mechanism of PAMS, it is yet to be proven if it shares the same pathophysiology as PLMS (Hoque
\& Chesson, 2010). However, it has been proposed that some possible lesions at the level of brain stem or spinal cord will result in the release of PAMS generators downstream (Chabli, Michaud, \& Montplaisir, 2000).

In our patient, apart from C3-7 cervical nerve root irritation and mild foraminal stenosis, there was no evidence of significant brainstem or spinal cord lesion. We also found it uncertain to correlate the patient's work-related repetitive arm movements with his PAMS symptoms.

Interestingly, there was a timing pattern in our patient's PAMS and PLMS, with the PAMS dominating at the first and PAMS+PLMS at the second half of his sleep. Similar to what we observed, one report has shown a temporal PAMS/PLMS relationship (Chabli, Michaud, \& Montplaisir, 2000). According to this study, arm movements preceded leg movements in $41 \%$ of cases during sleep and in 14\% upon wakefulness. Likewise, the cause and mechanism of this temporal link is yet to be explained (Chabli, Michaud, \& Montplaisir, 2000).

The presence of PAMS proposes that the structures accountable for generating this periodicity originate at more rostral levels in the neural axis and possibly involve 
the brainstem and other parts of the cortex. Research has also suggested that the presence of PAM upon wakefulness is noted in a considerable number of RLS patients. Moreover, patients with PAM during wakefulness might show more sleep disturbances (Michaud, Chabli, Lavigne, \& Montplaisir, 2000; Walters, 2007).

A wealth of evidence has implicated the central dopaminergic system and impaired iron processing both in RLS and PLMS. Meanwhile, it has remained unclear why both conditions predominantly involve the lower limbs (Torabi-Nami, 2012).

RLS involves leg paresthesias as well as motor restlessness and pacing, which leads to discomfort and difficulty in sleep onset. Symptoms are typically aggravated in the evening and at night, and transiently alleviated by movement. RLS and PLMS are shown to be strongly associated with each other. Another related phenomenon which needs more exploration is "arm restlessness." Of note, almost half of RLS patients are found to have arm restlessness as well. Individuals with arm restlessness tend to have more severe RLS and poorer sleep quality as compared to those without them (Michaud et al., 2000).

In conclusion, the treatment of PAMS has not been studied and requires more research. In our patient, despite several indexes confirming PAMS and PLMS, he did not recount symptoms of RLS. Patients with PAMS and restless arms appear to benefit from individualized treatment. It seems that until further research, physicians may use PLMS treatment approach to help their PAMS patients. To this end, the following set of clinically-supported measures are recommended (Torabi-Nami, 2012) :

1. Iron stores should be evaluated in those suspected of having PLMS/PAMS and a trial of oral iron therapy should be given in patients with iron deficiency.

2. Strict adherence to sleep hygiene needs to be emphasized.

3. First-line therapy would include a trial of a dopamine agonist/levodopa or dopamine agonists of the nonergoline class such as pramipexole. Other treatment options for intermittent symptoms include benzodiazepines and opioids.

4. Nonpharmacological therapy options include avoidance of aggravating drugs and substances such as caffeine and mental alerting activities.
5. PLMS and PAMS in the absence of insomnia or excessive daytime sleepiness do not seem to require treatment.

\section{Acknowledgments}

Authors would like to thank Ms Cheraghipour for her assistance. This study was funded by the School of Advanced Medical Sciences and Technologies and received supports from the Division of Pulmonology, Department of Internal Medicine, School of Medicine, Shiraz University of Medical Sciences.

\section{Conflicts of Interest}

Authors declared no conflict of interest.

\section{References}

Berry, R. B., Budhiraja, R., Gottlieb, D. J., Gozal, D., Iber, C., Kapur, V. K., et al. (2012). Rules for scoring respiratory events in sleep: Update of the 2007 AASM Manual for the Scoring of Sleep and Associated Events. Deliberations of the Sleep Apnea Definitions Task Force of the American Academy of Sleep Medicine. Journal of Clinical Sleep Medicine, 8(5), 597-619.

Chabli, A., Michaud, M., \& Montplaisir, J. (2000). Periodic arm movements in patients with the restless legs syndrome. European Neurology, 44(3), 133-138.

Grigg-Damberger, M. M. (2012). The AASM Scoring Manual four years later. Journal of Clinical Sleep Medicine, 8(3), 323-332.

Hoque, R., \& Chesson Jr, A. L. (2010). Pharmacologically induced/exacerbated restless legs syndrome, periodic limb movements of sleep, and REM behavior disorder/REM sleep without atonia: Literature review, qualitative scoring, and comparative analysis. Journal of Clinical Sleep Medicine, 6(1), 79-83.

Janssen, M. M., Harlaar, J., \& de Groot, I. J. (2015). Surface EMG to assess arm function in boys with DMD: A pilot study. Journal of Electromyography \& Kinesiology, 25(2), 323-328.

Karatas, M. (2007). Restless legs syndrome and periodic limb movements during sleep: Diagnosis and treatment. Neurologist, 13(5), 294-301.

Malangre, A., Leinen, P., \& Blischke, K. (2014). Sleep-related offline learning in a complex arm movement sequence. Journal of Human Kinetics, 40(1), 7-20.

Michaud, M., Chabli, A., Lavigne, G., \& Montplaisir, J. (2000). Arm restlessness in patients with restless legs syndrome. Movement Disorders, 15(2), 289-293.

Portaluppi, F., Cortelli, P., Buonaura, G. C., Smolensky, M. H., \& Fabbian, F. (2009). Do restless legs syndrome (RLS) and periodic limb movements of sleep (PLMS) play a role in nocturnal 
hypertension and increased cardiovascular risk of renally impaired patients? Chronobiology International, 26(6), 1206-1221.

Torabi-Nami, M. (2012). Neuroscience of Sleep and the Recent Advances in Diagnosis, Genetics and Treatment of Restless Legs Syndrome. Researcher, 4(3), 48-51.

Trotti, L. M., Bliwise, D. L., Greer, S. A., Sigurdsson, A. P., Gudmundsdottir, G. B., Wessel, T., et al. (2009). Correlates of PLMs variability over multiple nights and impact upon RLS diagnosis. Sleep Medicine, 10(6), 668-671.

Valko, P. O., Siccoli, M. M., \& Bassetti, C. L. (2009). Unilateral RLS with predominantly ipsilateral PLMS and variable response to dopaminergic drugs: A variant of idiopathic RLS? European Journal of Neurology, 16(3), 430-432.

Walters, A. S. (2007). Clinical identification of the simple sleeprelated movement disorders. Chest, 131(4), 1260-1266.

Walters, A. S., Lavigne, G., Hening, W., Picchietti, D. L., Allen, R P., Chokroverty, S., et al. (2007). The scoring of movements in sleep. Journal of Clinical Sleep Medicine, 3(2), 155-167.

Yokota, T., Shiojiri, T., \& Hirashima, F. (1995). Sleep-related periodic arm movement. Sleep, 18(8), 707-708.

Zucconi, M., Ferri, R., Allen, R., Baier, P. C., Bruni, O., Chokroverty, S., et al. (2006). The official World Association of Sleep Medicine (WASM) standards for recording and scoring periodic leg movements in sleep (PLMS) and wakefulness (PLMW) developed in collaboration with a task force from the International Restless Legs Syndrome Study Group (IRLSSG). Sleep Medicine, 7(2), 175-183. 